\title{
Uma nova espécie de Clelia (Serpentes, Colubridae) do sul do Brasil
}

\author{
Sérgio Augusto A. Morato ${ }^{1}$, Francisco L. Franco ${ }^{2}$ e Eros J. Sanches ${ }^{3}$ \\ ${ }^{1}$ Núcleo de Pesquisa e Extensão em Ciências Biológicas, Universidade Tuiuti do Paraná. Pós-graduação em Zoologia, \\ Universidade Federal do Paraná, e Laboratório de Herpetologia, Museu de História Natural Capão da Imbuia. R. Prof. \\ Benedito Conceição, 407, 82.810-080, Curitiba, Paraná, Brasil. E-mail: sergio.morato@utp.br. \\ ${ }^{2}$ Laboratório de Herpetologia, Instituto Butantan. Av. Vital Brazil, 1500, 05503-900, São Paulo, SP, Brasil. E-mail: \\ flfranco@butantan.gov.br. \\ ${ }^{3}$ Instituto de Pesquisa Científica e Conservacionista Criadouro de Cobras de Porto União. R. Frei Rogério, 257. 89400- \\ 000, Porto União, SC, Brasil.
}

\begin{abstract}
A new species of Clelia (Serpentes, Colubridae) from southern Brazil. Clelia hussami sp. nov. is described based on 10 specimens from southern Brazil. The new species can be diagnosed by the following arrangement of characters: 19 midbody dorsal rows, 7 supralabials and 8 infralabials, 204-225 ventrals, 47-56 paired subcaudals, ground coloration light brown with a fine dark reticulum in adults, presence of a narrow dark vertebral line and clear nucal collar more evident in juveniles, and a creamily light immaculated venter. A description of hemipenis is presented.
\end{abstract}

Keywords: Serpentes, Colubridae, Clelia hussami sp. nov., taxonomy, hemipenis, southern Brazil.

Palavras-chave: Serpentes, Colubridae, Clelia hussami sp. nov., taxonomia, hemipênis, sul do Brasil.

\section{Introdução}

O gênero Clelia Fitzinger, 1826 é composto por colubrídeos opistóglifos, de médio a grande porte, com pupila elíptica, escama cloacal única, escamas subcaudais em pares ou mistas e que, usualmente, apresentam variação ontogenética de colorido (Bailey 1967). Compreende as espécies Clelia bicolor (Peracca, 1904), C. clelia (Daudin, 1803), C. equatoriana

Recebido em 18 de agosto de 2003.

Aceito em 28 de novembro de 2003.

Distribuído em 19 de dezembro de 2003.
(Amaral, 1924), C. errabunda Underwood, 1993, C. montana Franco, Marques e Puorto, 1997, C. quimi Franco, Marques e Pourto, 1997, C. plumbea (Wied, 1820), C. rustica (Cope, 1878) e C. scytalina (Cope, 1867) (Zaher 1996, Franco et al. 1997). Ocorrem por quase toda a região Neotropical, desde o Sul do México até $42^{\circ} \mathrm{S}$ na Argentina (Scrocchi e Viñas 1990, Zaher 1996, Franco et al. 1997).

Recentemente, ocorreram importantes alterações taxonômicas envolvendo as espécies do gênero Clelia. Scrocchi e Viñas (1990) sinonimizaram Clelia occipitolutea (Duméril, Bibron e Duméril, 1854) com C. clelia. Zaher 
(1996) propôs o gênero Boiruna, distinguindoo de Clelia com base na morfologia do hemipênis e no padrão de colorido, para incluir B. maculata (Boulenger, 1896) e B. sertaneja Zaher, 1996. Zaher (1996) também demonstrou que o nome Brachyruton occiptoluteum Duméril, Bibron e Duméril, 1854, tradicionalmente atribuído ao gênero Clelia ( $C$. occiptolutea), é, de fato, um sinônimo júnior de Pseudoboa coronata Schneider, 1801, prevalecendo assim o nome específico Oxyrhopus maculatus Boulenger, 1896 para o táxon alocado no gênero Boiruna.

Zaher (1996) observou que os juvenis de Clelia rustica apresentam padrão de colorido semelhante ao dos adultos, exceto pela presença de um colar nucal claro, que desaparece rapidamente, de modo que os indivíduos com 500 mm de comprimento total já apresentam o padrão dos adultos. Franco et al. (1997) observaram que a presença de variação ontogenética de colorido em $C$. rustica nem sempre se verifica, salientando que esta diferença poderia sugerir a existência de duas espécies sob esse nome. A descoberta de novos exemplares com colar nucal claro e linha vertebral em diversos estágios do desenvolvimento ontogenético e sua associação a outros caracteres diagnósticos permitiu-nos reconhecer uma espécie de Clelia ainda não descrita, semelhante a $C$. rustica e $C$. montana. A descrição dessa nova espécie é o objetivo deste trabalho.

\section{Material e Métodos}

Foram examinados exemplares de Clelia das coleções herpetológicas Alphonse Richard Hoge, do Laboratório de Herpetologia do Instituto Butantan (IB, São Paulo, SP, Brasil), do Museu de História Natural Capão da Imbuia (MHNCI, Curitiba, PR, Brasil) e do Criadouro de Cobras de Porto União (CCPU, Porto União, $\mathrm{SC}$, Brasil). As observações foram feitas a olho nu ou com auxílio de microscópio estereoscópico. As medidas foram tomadas com escala graduada metálica com precisão de $1 \mathrm{~mm}$ e com paquímetro com precisão de $0,1 \mathrm{~mm}$.

As contagens e medidas de escamas e placas cefálicas foram feitas seguindo o proposto por Dowling (1951) e Peters (1964). Contagens e medidas de estruturas pares são apresentadas na ordem lado direito / lado esquerdo do corpo; quando um único valor é apresentado, refere-se ao lado direito do corpo. A terminologia empregada na descrição do hemipênis segue Dowling e Savage (1960), Myers e Cadle (1994) e Zaher (1999).

Clelia hussami sp. nov. (Figura 1)

Clelia rustica (part.), Zaher, 1996. Boll. Mus. Reg. Sci. Nat. Torino 14(2): 312-4.

Clelia rustica (part.), Franco, Marques e Puorto, 1997. J. Herpetol., 31(4): 483-90.

Holótipo: IB 32859, fêmea sub-adulta, procedente do município de Cruz Machado (26ํำ'S, 51²0'W, 940 m), estado do Paraná, Brasil. Entregue ao Instituto Butantan por Lourenço Miynarczk em 9 de fevereiro de 1972.

Parátipos: IB 4544, fêmea jovem, procedente do município de Rio das Antas (265's, $51^{\circ} 04^{\prime} \mathrm{W}, 830 \mathrm{~m}$ ), estado de Santa Catarina, Brasil, entregue ao Instituto Butantan por Richard Weigert, em 17 de outubro de 1928; IB 15692 , macho adulto, procedente do município de Ipoméia (26 56' S, 5106' W, 830 m), estado de Santa, Catarina, Brasil, entregue ao Instituto Butantan por Jorge Moresco, sem data determinada; IB 15759, fêmea adulta, sem crânio, procedente do município de Rio Azul (254' S, $50^{\circ} 47^{\prime} \mathrm{W}, 925 \mathrm{~m}$ ), estado do Paraná, Brasil, entregue ao Instituto Butantan por Gabriel Rynsza, em 14 de novembro de 1953; MHNCI 6576, fêmea jovem, procedente do município de Porto União (26 $\left.{ }^{\circ} 14^{\prime} \mathrm{S}, 51^{\circ} 04^{\prime} \mathrm{W}, 795 \mathrm{~m}\right)$, estado do Paraná, Brasil, coletado por Eros J. Sanches e Clovis R. Gurski, em 15 de dezembro de 1993; MHNCI 11402, macho adulto, procedente do município de Bituruna (2609'S, 51³3’W, 987 


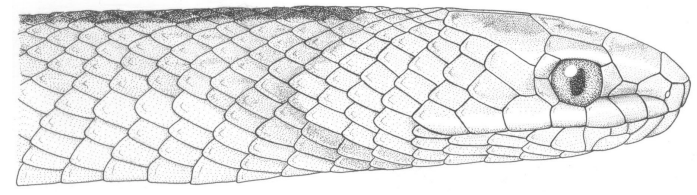

em 2 de janeiro de 1952; CCPU 712, macho filhote, procedente do município de Fraiburgo ( $27^{\circ} 01^{\prime} \mathrm{S}, 5^{\circ} 55^{\prime} \mathrm{W}, 1048 \mathrm{~m}$ ), estado de Santa Catarina, Brasil, coletado em setembro de 1996 por Marco Antonio Dias da Silva; CCPU 1180, fêmea adulta, procedente do município de Irineópolis $\left(26^{\circ} 14^{\prime} \mathrm{S}, 50^{\circ} 48^{\prime} \mathrm{W}, 762 \mathrm{~m}\right)$, estado de Santa Catarina, Brasil, coletado em 11 de

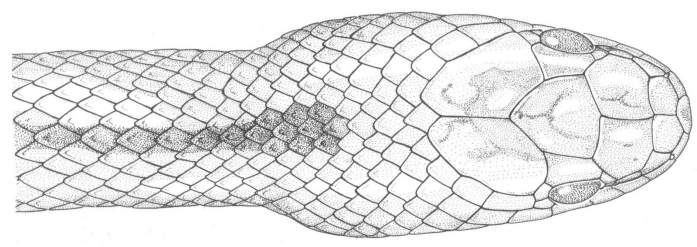
dezembro de 2001 por Mauri Giovan Bianco.

Distribuição geográfica: Os exemplares de Clelia hussami procedem da porção central do Planalto Meridional Brasileiro, em área de altitude superior a $700 \mathrm{~m}$. A região é caracterizada pelo tipo climático $\mathrm{Cfb}$ de Köppen, sendo área de distribuição restrita das florestas com araucária e campos limpos associados (Figura 2). À semelhança de outras espécies de serpen-

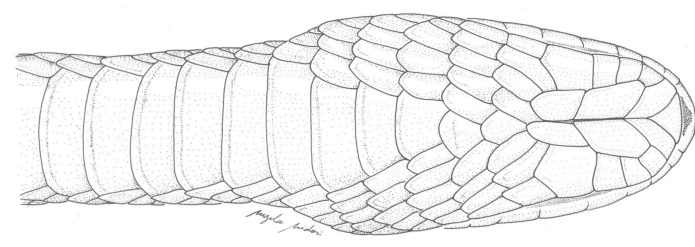
tes (e.g., Bothrops cotiara, Müller 1973; Pseudoboa haasi, Morato et al. 1995), C. hussami pode tratar-se de espécie endêmica da região de domínio das florestas com araucária da porção sul do Brasil, não sendo, contudo, restrita a ecossistemas florestais propriamente ditos.

Figura 1 - Holótipo de Clelia hussami sp. nov. (IB 32859) em vistas lateral (A), dorsal (B) e ventral (C). Barra de escala $=1 \mathrm{~cm}$.

m), estado do Paraná, Brasil, coletado em agosto de 1997 por estudantes do Colégio Estadual Santa Bárbara; MHNCI 11403, fêmea jovem, procedente do município de Caçador (26 $44^{\prime} \mathrm{S}$, $\left.51^{\circ} 02^{\prime} \mathrm{W}, 800 \mathrm{~m}\right)$, estado de Santa Catarina, Brasil, coletado em 18 de fevereiro de 1999 por Giorgi Luy Burlim.

Outros exemplares analisados: IB 14979, macho adulto, ressecado e descorado, procedente do município de Caçador $\left(26^{\circ} 44^{\prime} \mathrm{S}, 5^{\circ} 02^{\prime} \mathrm{W}\right.$, $800 \mathrm{~m}$ ), estado de Santa Catarina, Brasil, entregue ao Instituto Butantan por Francisco Lima

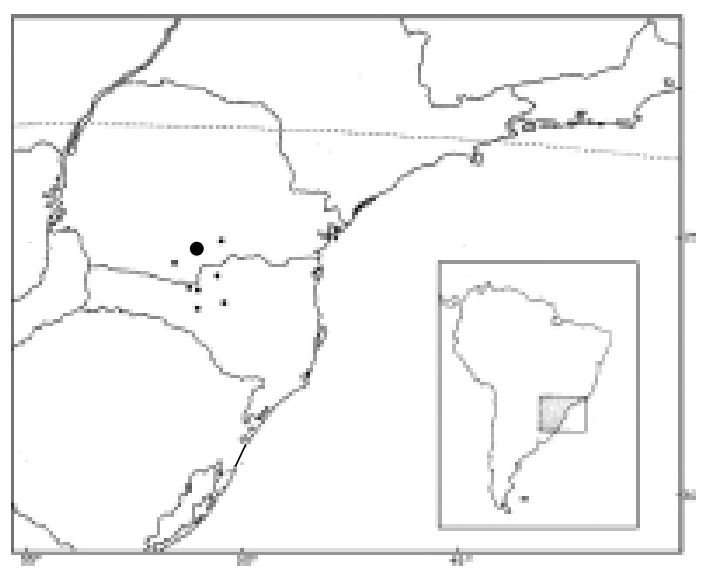

Figura 2 - Distribuição geográfica de Clelia hussami sp. nov. O ponto maior identifica a localidadetipo, município de Cruz Machado, Paraná, Brasil. 
Diagnose: Clelia hussami distingue-se de $C$. equatoriana, C. errabunda e C. scytalina por apresentar 19 fileiras de escamas dorsais no meio do corpo (Zaher 1996), 17 naquelas espécies. Clelia hussami apresenta menos de 56 pares de escamas subcaudais, adultos com dorso castanho-claro marcado com tênue reticulado escuro nas escamas dorsais, fina linha vertebral escura com cerca de duas fileiras de escamas de largura e colar nucal claro inconspícuos; juvenis vermelhos com linha vertebral castanho-escura fina, colar nucal esbranquiçado e cabeça castanho-escura. Clelia clelia e C. plumbea possuem 70 ou mais pares de escamas subcaudais, dorso homogêneo cinza-escuro ou negro nos adultos e uniformemente avermelhado com cabeça negra e colar branco nos juvenis (Zaher 1996). Clelia hussami tem 7 supralabiais, $8 \mathrm{em}$ C. quimi, C. montana e C. bicolor (Franco et al. 1997). Clelia hussami apresenta 47 a 50 pares de escamas subcaudais em fêmeas e 49 a 56 pares em machos, enquanto $C$. quimi apresenta 58 pares ou mais e $C$. bicolor tem 53 a 62 pares em fêmeas e 64 a 73 pares em machos (Franco et al. 1997). Clelia hussami possui supralabiais imaculadas, enquanto $C$. montana $e \quad C$. rustica apresentam supralabiais pigmentadas. Clelia hussami exibe uma faixa dorsal com duas a três fileiras de escamas dorsais, tênue nos adultos, enquanto $C$. montana apresenta uma faixa escura dorsal com três a sete fileiras, evidente nos adultos (Franco et al. 1997). Clelia hussami tem 8 infralabiais, ventre imaculado, adultos com dorso castanho claro marcado com tênue reticulado escuro nas escamas dorsais, fina linha vertebral escura, colar nucal claro inconspícuo e juvenis avermelhados com linha vertebral escura fina, colar nucal esbranquiçado e cabeça marrom-escura. Clelia rustica possui normalmente 9 infralabiais, dorso marrom com reticulado escuro evidente e ventre de fundo creme com manchas escuras nos adultos e juvenis, sendo que estes últimos podem apresentar um colar nucal claro que é perdido pouco tempo após o nascimento (Zaher 1996, Franco et al. 1997).
Descrição do Holótipo: fêmea sub-adulta, com comprimento rostro-cloacal de $520 \mathrm{~mm}$, comprimento da cauda de $82 \mathrm{~mm}$ e comprimento da cabeça de $17,7 \mathrm{~mm}$. Cauda relativamente curta (13,6\% do comprimento total), corpo levemente deprimido, com constrição cervical pouco pronunciada, e focinho arredondado.

Rostral bem visível em vista dorsal, um pouco mais larga que alta. Duas internasais pequenas, menores que a metade das pré-frontais. Duas pré-frontais. Frontal hexagonal, um pouco mais longa que larga $(3,8 \mathrm{~mm} \times 4,0 \mathrm{~mm})$; distância da frontal à tangente à rostral $(3,8 \mathrm{~mm})$ semelhante ao comprimento da frontal. Duas parietais $(5,5 \mathrm{~mm}$ de comprimento $\mathrm{x} 3,7 \mathrm{~mm}$ de largura) menores que a menor distância que as separam da tangente à rostral $(6,6 \mathrm{~mm})$. Nasal dividida. Loreal pequena, mais longa que alta. Uma pré-ocular e duas pós-oculares de ambos os lados. Pós-ocular superior de dimensões semelhantes às da inferior. Temporais $2+3 / 2+3$. Supralabiais $7 / 7,3^{\mathrm{a}}$ e $4^{\mathrm{a}}$ em contato com o olho. Infralabiais $8 / 8,1^{\text {a }}$ a $5^{\mathrm{a}}$ em contato com mentonianas anteriores e $5^{\mathrm{a}}$ em contato com mentonianas posteriores. Mentoniana anterior maior que posterior, com 3,3 e 2,9 mm, respectivamente. Cinco fileiras de escamas gulares separam a última infralabial da $1^{\text {a }}$ ventral. Olho com pupila vertical elíptica, diâmetro horizontal de 2,5 $\mathrm{mm}$, menor que a distância que o separa da linha imaginária que tangencia perpendicularmente a margem anterior da escama rostral $(4,4$ $\mathrm{mm}$ ); diâmetro vertical do olho de $2,0 \mathrm{~mm}$, aproximadamente o dobro da menor distância que o separa da abertura bucal $(1,1 \mathrm{~mm})$.

Escamas dorsais lisas, com duas fossetas apicilares, em 19/19/17 fileiras. A redução se dá pela soma da $3^{\mathrm{a}}$ e $4^{\mathrm{a}}$ fileiras na altura da escama ventral 147 do lado direito e 150 do lado esquerdo. Três pré-ventrais e 212 ventrais. Escama cloacal única. 48 pares de escamas subcaudais. Escudo terminal presente.

Coloração em álcool. Exemplar um pouco descorado pela preservação, porém mantendo o padrão de desenho bem visível. Dorso da cabe- 
ça marrom escuro, quase negro. Um colar nucal claro ocupa o extremo posterior das parietais, três escamas dorsais de comprimento e a região temporal. Após o colar claro, ocorre uma mancha escura expandida transversalmente, de onde sai uma linha vertebral escura até a cauda, inclusive, que ocupa a fileira vertebral e a metade de cada escama paravertebral adjacente. Escamas dorsais com leve reticulado escuro. Supralabiais claras, sendo que as anteriores ao olho apresentam pequena porção de seus ápices com a coloração escurecida do dorso da cabeça. Região gular e ventre imaculados, brancoamarelados; apenas as bordas adjacentes à primeira fileira de escamas dorsais apresenta a coloração escura do dorso. Região subcaudal clara, com poucas manchas escuras esparsas.

Variação. Em machos, o comprimento rostrocloacal variou de 265 a $762 \mathrm{~mm}(\mathrm{~N}=4)$, o comprimento da cauda variou de 46 a $160 \mathrm{~mm}(\mathrm{~N}$ $=4$ ), representando cerca de $17 \%$ do comprimento total. $\mathrm{O}$ comprimento da cabeça variou de 12,3 a 26,2 mm $(\mathrm{N}=4)$. Em fêmeas, o comprimento rostro-cloacal variou de 233 a 1080 $\mathrm{mm}(\mathrm{N}=6)$ e o comprimento da cauda variou de 40 a $168 \mathrm{~mm}(\mathrm{~N}=6)$, representando cerca de $13,8 \%$ do comprimento total. O comprimento da cabeça variou de 11,9 a $32,4 \mathrm{~mm}(\mathrm{~N}=6)$. A forma geral do corpo e a escutelação cefálica seguem o descrito para o holótipo. Nos exemplares CCPU 1180 e IB 14979, os pré-frontais tocam as supralabiais pela ausência de loreais de ambos os lados. No espécime CCPU 712, a temporal secundária superior é fundida à temporal secundária central do lado direito, resultando na fórmula temporal $2+2 / 2+3$. O espécime IB 15759 apresenta 7/8 infralabiais; o espécime IB 4544 apresenta as primeiras 4 infralabiais em contato com as mentonianas anteriores e a $4^{\mathrm{a}}$ e $5^{\mathrm{a}}$ em contato com as posteriores. O exemplar MHNCI 11403 apresenta 4 fileiras de gulares separando a última infralabial da $1^{\text {a }}$ ventral. A posição reducional afetando as $3^{\mathrm{a}}$ e $4^{\mathrm{a}}$ fileiras de dorsais variou da ventral 138 , no exemplar IB 15692, até a ventral 162, no espécime MHNCI 11403. Há dimorfismo sexual em relação ao número de ventrais, maior nas fêmeas. As ventrais variam de 204 a 210 (média de 206,7; $\mathrm{N}=4$ ) nos machos e de 212 a 225 (média de 218,0; $\mathrm{N}=6$ ) nas fêmeas. O dimorfismo sexual nas subcaudais é pouco pronunciado, sendo que os machos apresentam números médios superiores aos das fêmeas. As subcaudais variam de 49 a 56 (média de 53,5; N =4) nos machos e de 47 a 50 (média de 49,1; $\mathrm{N}=6$ ) nas fêmeas.

Variação ontogenética de colorido. Os filhotes são claros dorsalmente, com cabeça negra. A coloração negra cobre totalmente as placas cefálicas dorsais e segue-se um colar nucal branco de largura equivalente a três escamas dorsais, que desce lateralmente até a região paraventral. Após o colar, há uma macha escura expandida transversalmente de onde parte uma fina linha vertebral castanho-escura, quase negra, que, tomando a fileira vertebral e meia a uma escama paravertebral de cada lado, atinge a cauda, onde se torna mais delgada. Na medida em que aumenta o tamanho dos indivíduos, aumenta a intensidade de um pigmento castanho, escurecendo pouco a pouco o dorso, que, gradativamente, revela um fino reticulado escuro nas bordas das escamas dorsais. O colar claro e a linha escura dorsal ficam gradativamente mais inconspícuos, sendo, porém, ainda visíveis no maior exemplar (IB 15759, comprimento total de $1248 \mathrm{~mm}$ ). O ventre é claro, imaculado, não apresentando variação ontogenética.

O exemplar IB 14979 está ressecado e descorado, não apresentando a linha vertebral escura e o colar nucal claro; porém, apresenta outras características da espécie, como ventre e supralabiais imaculados e retículo escuro dorsal pouco evidente. Destaca-se que tanto a perda da linha dorsal e do colar nucal como o retículo pouco evidente e o ventre e as supralabiais imaculados podem ser artefatos da descoloração (ver Discussão).

Em vida, o exemplar MHNCI 6576, um filhote, apresentou colorido de fundo vermelho e 
ventre branco amarelado. O exemplar CCPU 1180, uma fêmea adulta, apresentou cor castanho-cobreado e o reticulado e a linha dorsal muito sutis.

Hemipênis. A descrição a seguir baseia-se no hemipênis direito do exemplar IB 15692, que se encontra desidratado. Hemipênis bilobado, bicapitado, com sulco espermático centrífugo, que se divide na região mediana do corpo do hemipênis e se estende até a porção mediana de cada capítulo. Lobos divergentes, 1,5 vez mais longos que largos, densamente ornamentados por papilas na porção distal, maiores na porção apical de cada capítulo. Face sulcada, com espinhos grandes na porção mediana do corpo do hemipênis dispostos em posição distal e paralela ao sulco espermático; superfície intra-sulcar lisa, com dois grandes espinhos na base de cada um dos capítulos. Face assulcada coberta por pequenos espinhos na região mediana do corpo do hemipênis, tornando-se lisa na porção apical entre os capítulos. Superfície lateral com espinhos medianos a grandes dispostos em duas fileiras presentes em cada um dos lados do hemipênis.

Etimologia: o epíteto específico hussami é uma homenagem a Hussam El Dine Zaher, do Museu de Zoologia da Universidade de São Paulo, São Paulo, SP.

\section{Discussão}

Franco et al. (1997) notaram a variação de colorido dos juvenis de Clelia rustica e levantaram a hipótese de que essa variação pode estar relacionada à existência de mais de uma espécie sob este nome. Com a localização de mais exemplares com padrão lineado, foi possível reavaliar os dados morfológicos e distinguir $C$. rustica de $C$. hussami. O número de infralabiais de $C$. rustica é mais constante do que Franco et al. (1997) apresentaram, pois cinco exemplares citados com 8 infralabiais, de fato correspondem a C. hussami (IB 4544, 15692, 15759, 32859,
14979). Desse modo, quase todos os espécimes de $C$. rustica apresentam 9 infralabiais, raramente 8 (um com $8 / 8$ e um com 9/8). A grande semelhança morfológica entre $C$. rustica e $C$. hussami foi responsável pela inclusão de exemplares desta última espécie junto aos de $C$. rustica nos trabalhos de Zaher (1996) e Franco et al. (1997). Essa semelhança é ainda mais acentuada com $C$. montana, porque os juvenis apresentam, adicionalmente, padrão semelhante de colorido (ver fotografia em Marques, Eterovic e Sazima 2001) e mesmo número de supra e infralabiais.

Segundo Zaher (1994, 1999) o gênero Clelia, como atualmente definido, é polifilético, pois $C$. quimi, $C$. bicolor e $C$. montana formariam um grupo monofilético relacionado ao clado composto por Oxyrhopus Wagler, 1830 e Siphlophis Fitzinger, 1843. Zaher (1999) postulou que o gênero Clelia pode ser dividido em dois grupos com base na morfologia dos hemipênis. Um grupo incluiria $C$. bicolor, $C$. quimi e $C$. montana e outro, todas as demais espécies conhecidas à época. O primeiro grupo foi caracterizado por seus integrantes compartilharem hemipênis bicaliculado e bicapitado, com região intrasulcar portando uma fileira de três espinhos de cada lado (duas ou três em $C$. montana). Nessas três espécies, não há cálices no corpo (body calyces) e bolsas caliculares (calicular pockets) na superfície assulcada medial dos lobos e da região da bifurcação do hemipênis (lobular crotch). O segundo grupo é caracterizado por possuir hemipênis bicaliculados e bicapitados, com sulco espermático centrífugo, com um ou dois espinhos intrasulcares, e duas ou três fileiras de grandes espinhos laterais, com exceção de $C$. plumbea, que não possui espinhos ou espínulos. Todas as espécies desse segundo grupo possuem um par de bolsas caliculares bem-desenvolvidas e cálices no corpo pouco desenvolvidos nas superfícies medial e assulcada dos lobos. Se o grupo composto por $C$. quimi, C. bicolor e $C$. montana for excluído do gênero Clelia, é possível que C. hussami seja alocada juntamente 
com essas espécies devido a sua grande semelhança morfológica e hemipeniana com $C$. montana (em especial quanto à presença de fileiras laterais de grandes espinhos e grande número de papilas nos capítulos), o que pode ser indicativo de proximidade filogenética.

O padrão de distribuição disjunta entre populações e espécies filogeneticamente aparentadas ocorrentes nas regiões de abrangência da floresta com araucária da região sul do Brasil (área de distribuição de $C$. hussami) e das porções elevadas presentes ao sul do estado do Rio de Janeiro, sudeste de Minas Gerais e extremo nordeste de São Paulo (área de distribuição de C. montana) é observado em outras espécies de serpentes e de outros grupos animais. Por exemplo, temos os pares de espécies Bothrops cotiara - B. fonsecai (Viperidae), Pseudoboa haasi - P. serrana (Colubridae; Morato et al. 1995) e Lepidocolaptes falcinellus - L. squamatus (Aves, Dendrocolaptidae; Silva e Straube 1996) e a distribuição disjunta de Ptychophis flavovirgatus (Porto e Caramaschi 1988). Essas ocorrências podem ser explicadas por dados paleoambientais que mostram que, na passagem do Pleniglacial Médio (60.000 a 28.000 anos AP) a Superior (28.000 a 13.000 anos AP), desde cerca de 36.000 anos AP, as florestas com araucária estendiam-se até o Distrito Federal, no Planalto Central Brasileiro (Barberi 2001). A regressão dessas florestas e sua fragmentação nos moldes atuais podem ter influenciado as distribuições descontínuas e as especiações citadas acima. Desse modo, pode-se esperar que outras espécies de serpentes e outros grupos animais e vegetais, tanto ocorrentes em áreas florestais como nos campos limpos associados às florestas com araucária (a exemplo de Ditaxodon taeniatus, Colubridae; R. A. Thomas e colaboradores, dados não publicados) venham corroborar esse padrão. É importante ressalvar que a distribuição de espécies campestres pode ter sofrido influências paleoambientais anteriores ao das espécies florestais (e.g., Klein 1975, Behling 1998).

Franco et al. (1997) agruparam as espécies de Clelia com 19 fileiras de escamas dorsais com base em semelhanças primárias, alocando C. montana e C. rustica em um mesmo grupo, por compartilharem hemipênis com lobos divergentes e de comprimento e largura semelhantes. O grupo formado por C. clelia, C. bicolor e $C$. quimi possui lobos convergentes ou paralelos, com largura máxima de aproximadamente metade de seu comprimento (Franco et al. 1997). Clelia hussami compartilha características do hemipênis com o grupo de $C$. montana e $C$. rustica, fato que, reforçado pela semelhança morfológica entre as espécies, sugere cautela quanto ao seu relacionamento filogenético, sendo prudente aguardar os trabalhos sistemáticos de Zaher para avaliar novamente a situação.

O exemplar IB 14979 foi excluído da série tipo, pois não apresenta a linha vertebral escura e o colar nucal claro. Considerando os outros caracteres, não há o que impeça de identificálo como C. hussami: apresenta 7 supralabiais e 8 infralabiais, ventre e supralabiais imaculados, reticulado escuro dorsal inconspícuo e contagens de ventrais $2+210$, dorsais 19/19/17 e subcaudais 54/54, de acordo com a espécie. Esse espécime está ressecado e perdeu muito de sua cor original. Assim, identificamos provisoriamente o exemplar IB 14979 como $C$. hussami, mas o excluímos da série-tipo. Os exemplares CCPU 712 e CCPU 1180 não foram incluídos na série-tipo por estarem depositados em uma coleção nova e regional.

\section{Agradecimentos}

Agradecemos a Júlio César de Moura-Leite a leitura crítica do manuscrito e o empréstimo dos exemplares da Coleção Herpetológica do $\mathrm{Mu}$ seu de História Natural Capão da Imbuia, a Valdir José Germano o apoio na curadoria da Coleção Herpetológica Alphonse Richard Hoge (Instituto Butantan) e a dois revisores anônimos as contribuições. Agradecemos a Ângela Midori o desenho da Figura 1 e à FAPESP e à Fundação O Boticário de Proteção à Natureza os subsídios financeiros concedidos respectivamente a F. L. Franco e S. A. A. Morato. 


\section{Referências Bibliográficas}

Bailey, J. R. 1967. The synthetic approach to colubrid classification. Herpetologica 23: 155-161.

Barbieri, M. 2001. Mudanças paleoambientais na região dos cerrados do Planalto Central durante o Quaternário tardio: Estudo da Lagoa Bonita, DF. Tese de Doutorado Não-publicada, Universidade de São Paulo, Brasil.

Behling, H. 1998. Late quaternary vegetational and climatic changes in Brazil. Review of Palaeobotany and Palynology 99:143-156.

Dowling, H. G. 1951. A proposed standard system of counting ventral in snakes. British Journal of Herpetology 1: 97-99.

Dowling, H. G. e J. M. Savage. 1960. A guide to the snake hemipenis: a survey of basic structure and systematic characteristics. Zoologica 45: 17-28

Franco, F. L., O. A. V. Marques e G. Puorto. 1997. Two new species of colubrid snakes of the genus Clelia from Brazil. Journal of Herpetology 31: 483-490.

Klein, R.M. 1975. Southern Brazilian phytogeographic features and the probable influence of Upper Quaternary climatic changes in the floristic distribution. Boletim Paranaense de Geociências 33: 67-88.

Marques, O. A. V., A. Eterovic e I. Sazima. 2001. Serpentes da Mata Atlântica - guia ilustrado para a Serra do Mar. Ribeirão Preto. Holos. 184 pp.

Morato, S. A. A., J. C. Moura-Leite, A. L. C. Prudente e R. S. Bérnils. 1995. A new species of Pseudoboa Schneider, 1801 from Southeastern Brazil (Serpentes: Colubridae: Xenodontinae: Pseudoboini). Biociências 3: $253-264$

Müller, P. 1973. The dispersal centres of terrestrial vertebrates in the Neotropical Realm. Biogeographica 2: $1-250$
Myers, C. W. e J. E. Cadle. 1994. A new genus for South American snakes related to Rhadinaea obtusa (Colubridae) and resurrection of Taeniophallus Cope for the Rhadinaea brevirostris group. American Museum Novitates 3102: 1-33.

Peters, J. A. 1964. Dictionary of Herpetology - a brief and meaningful definition of words and terms used in herpetology. New York. Hafner Publishing Company. $392 \mathrm{pp}$.

Porto, M. e U. Caramaschi. 1988. Notes on taxonomic status, biology and distribution of Ptychophis flavovirgatus Gomes, 1915 (Ophidia, Colubridae). Anais da Academia Brasileira de Ciências 60: 471475.

Scrocchi, G. e M. Viñas. 1990. El gênero Clelia (Serpentes: Colubridae) em la República Argentina: revisión y comentários. Bollettino del Museo Rerionale di Scienze Naturali - Torino 8: 487-499.

Silva, J. M. C. e F. C. Straube. 1996 Systematics and biogeography of scaled woodcreepers (Aves: Dendrocolaptidae). Studies on Neotropical Fauna and Environment 31: 3-10.

Zaher, H. 1994. Phylogénie des Pseudoboini et évolution des Xenodontinae sud-américans (Serpentes, Colubridae). Tese de Doutorado Não-publicada. Musée National d'Histoire Naturelle, Paris, França.

Zaher, H. 1996. A new genus and species of pseudoboine snake, with a revision of genus Clelia (Serpentes, Xenodontinae). Bollettino del Museo Rerionale di Scienze Naturali, Torino 14: 289-337.

Zaher, H. 1999. Hemipenial morphology of the South American xenodontine snakes, with a proposal for a monophyletic Xenodontinae and reappraisal of colubroid hemipenes. Bulletin of the American Museum of Natural History 240: 1-168. 